\section{Long standing eculizumab treatment without anticoagulant therapy in high-risk thrombogenic paroxysmal nocturnal hemoglobinuria}

\author{
Hassan A. Al-Jafar, Salma M. AIDallal, \\ Haifa A. Askar, Ali M. Aljeraiwi, \\ Ahmad Al-Alansari \\ Department of Hematology, Amiri \\ Hospital, Kuwait City, Kuwait
}

\begin{abstract}
Paroxysmal nocturnal hemoglobinuria (PNH) is an ultra-orphan disease affecting all hematopoietic cell types. The abnormality of red blood cells in this disease predisposes to intravascular complement-mediated hemolysis. Eculizumab is an orphan drug used to treat this rare disease. Thrombosis is the key cause of death in PNH patients in about $40 \%$ to $67 \%$ of cases. We report the case of a woman presenting with PNH complicated with serious Budd-Chiari syndrome thrombosis and with a stent inserted in the portal vein. She refused to take any anticoagulant treatment since she commenced eculizumab 4 years before. No thrombotic events happened since that time. This case could add an extra benefit for eculizumab, which could be used as an antithromboembolic prophylactic agent in $\mathrm{PNH}$, especially in patients with thrombocytopenia, where the use of anticoagulant agents is extremely hazardous. More randomized studies might establish the use of eculizumab without anticoagulants to avoid serious bleeding that could happen in thrombocytopenic PNH patients.
\end{abstract}

\section{Introduction}

Paroxysmal nocturnal hemoglobinuria (PNH) is an ultra-orphan disease. It is a disorder of hematopoietic stem cell that affects all cell lineages. ${ }^{1}$ The term ultra-orphan disease describes in United Kingdom the conditions with a disease prevalence of $<1$ case per 50,000 population, ${ }^{2}$ while $\mathrm{PNH}$ incidence is $1-2$ cases per million. The abnormality of the red blood cells (RBCs) in PNH predisposes them to intravascular complement-mediated hemolysis. ${ }^{3}$ The disorder is caused by somatic mutation in the X-linked phosphatidyl-inositol glycans class A $(P I G-A)$ gene. $P I G-A$ gene product is needed for biosynthesis of glycosyl-phos- phatidyl-inositol anchor (GPA), a glycolipid moiety that adheres many proteins to the plasma membrane of the cells, on the membrane of the RBCs. ${ }^{4} \mathrm{~A}$ large number of membrane protein deficiencies have been found in $\mathrm{PNH}$, specifically the complement regulatory proteins CD55 and CD59. ${ }^{5}$ The absence of these proteins are fundamental to the pathophysiology of the disease. CD55 inhibits C3 convertase and CD59 block the formation of the membrane attack complex (MAC) by inhibiting incorporation of $\mathrm{C} 9$ into the MAC. The loss of these regulatory proteins make RBCs more susceptible to both intravascular and extravascular hemolysis, but it is the intravascular hemolysis that contributes to the morbidity and mortality from in the disease. ${ }^{6}$ In PNH, failure to regulate complement on the erythrocyte membrane can lead to massive intravascular hemolysis. Free hemoglobin has enormous affinity for nitric oxide (NO) and can severely deplete its plasma level to the point of causing symptoms; it could manifest clinically as fatigue, abdominal pain, esophageal spasm, hemoglobinuria, and possibly thrombosis. ${ }^{7}$

PNH is variable, ranging from indolent to life threatening disease with a median survival rate of 10 to 15 years. Thrombosis is the key cause of death in PNH patients in about $40 \%$ to $67 \%$ of cases. $^{8}$

Venous thrombosis can occur anywhere, including hepatic splenic, portal and cerebral veins. ${ }^{9}$ However, others may die of complications, such as bone marrow failure, meyelodysplastic syndrome, renal failure and leukemia. ${ }^{10}$ Hepatic vein thrombosis is documented as one of the most common sites of thrombosis affecting PNH patients. ${ }^{11}$ Superior sagittal sinus thrombosis is the most common neurologic complication. ${ }^{12}$

The diagnosis of PNH is often difficult because the symptoms are often multifaceted and hemoglobinuria is absent in around 25\% of cases. PNH is suspected in patients showing mild to severe anemia with moderate reticulocytosis, raised serum lactate dehydrogenase (LDH) and possibly mild jaundice, with negative Coombs tests. ${ }^{13}$ Conventional treatment of $\mathrm{PNH}$ involves mainly supportive measures aiming to control the clinical manifestations of the disease such as anticoagulants and blood transfusion. Anticoagulants are the most common way to treat blood clots in patients with PNH and are usually administered on regular basis. Yet, anticoagulants administration can be challenging, because PNH patients often have low platelet counts, and the international normalized ratio (INR) is extremely hazardous to be maintained in therapeutic range in severely thrombocytopenic PNH patients. ${ }^{14}$

Eculizumab is a newly developed humanized monoclonal antibody (mAB) derived from the murine anti-human $\mathrm{C} 5 \mathrm{mAB}$. The $\mathrm{mAb}$ precisely binds the terminal complement fraction 5,
Correspondence: Hassan A. Al-Jafar, Department of Hematology, Amiri Hospital, 25, Kuwait City, Kuwait.

Tel.: +965.2245.0005.

E-mail: cbc9@hotmail.com

Key words: Paroxysmal nocturnal hemoglobinuria; eculizumab; thrombosis; orphan; anticoagulant.

Acknowledgments: the authors thank Mr Yaser Al Yacoub the senior specialist in immunophenotyping laboratory for his work on this patients samples and for his precious advices. The authors also thank Mr Yousef Al-Jafar for English language revision and editing of manuscript.

Contributions: the authors contributed equally.

Conflict of interest: the authors declare no potential conflict of interest.

Conference presentation: this case was presented in the poster day of the faculty of medicine, the Kuwait University; May 2015.

Received for publication: 26 March 2015

Revision received: 13 August 2015.

Accepted for publication: 2 September 2015.

This work is licensed under a Creative Commons Attribution NonCommercial 3.0 License (CC BYNC 3.0).

(C) Copyright H.A. Al-Jafar et al., 2015

Licensee PAGEPress, Italy

Hematology Reports 2015; 7:5927

doi:10.4081/hr.2015.5927

thus inhibiting the cleavage to $\mathrm{C} 5 \mathrm{a}$ and $\mathrm{C} 5 \mathrm{~b}$, therefore blocking the formation of MAC, which is the terminal effector mechanism lead to intravascular hemolysis of PNH RBCs. Eculizumab has found to be highly efficient in reducing intravascular hemolysis; treatment with eculizumab cuts the need for blood transfusions, reduced risks of thrombosis, and improves quality of life. ${ }^{15}$ Although this patient has a BCS, portal vein stent and continuous high risk of thrombosis, she refused to take any anticoagulant since she started eculizumab over the last 4 years. Up to date there is no established guideline to use eculizumab without anticoagulants despite its protective effect in PNH. Eculizumab could be a lifesaving drug when PNH is complicated by sever thrombocytopenia which is a common complication of this disease.

\section{Case Report}

In 2006, a 39-year-old female was seen by the hepatologist because of abdominal pain, 
Table 1. The improvements after Eculizumab.

\begin{tabular}{lcccccccc} 
Parameter & 2006 & 2007 & 2010 & 2011 & 2012 & 2013 & 2014 & 2015 \\
White blood cells, $10^{9} / \mathrm{L}$ & 2.5 & 2.9 & 1.9 & 4.3 & 9.7 & 2.5 & 2.9 & 2.6 \\
Hemoglobin, g/L & 8.4 & 9.5 & 7.5 & 8.7 & 9.5 & 10.5 & 10.7 & 10.4 \\
\hline Platelets, $10^{9} / \mathrm{L}$ & 72 & 94 & 63 & 44 & 113 & 63 & 72 & 78 \\
Reticulocytes, $\%$ & $\mathrm{NA}$ & $\mathrm{NA}$ & 5.5 & 6.72 & 4.22 & 3.94 & 5.24 & 6.42 \\
\hline Alanine-aminotransferase, U/L & 10 & 20 & 17 & 11 & 16 & 11 & 12 & 14 \\
Aspartate-aminotransferase (10-42 U/L) & 20 & 43 & 107 & 24 & 25 & 23 & 20 \\
\hline Lactate dehydrogenase (90-248 U/L) & $\mathrm{NA}$ & $\mathrm{NA}$ & 1541 & 288 & 235 & 289 & 265 & 231 \\
\hline
\end{tabular}

NA, not available.

hepatosplenomegaly and thrombocytopenia. Abdominal Doppler ultrasound and CT abdomen revealed BCS. She was put on oral anticoagulant Coumadin to maintain INR between 2-3. She was suspected to have seronegative systemic lupus erythromatous or seronegative APL. Hydroxychloroquine was also commenced, based on her thrombocytopenia and BCS thrombosis. With trans-jugular intrahepatic portacaval shunt a hepatic vein stent (TIPS) was successfully placed. The results of laboratory tests were as follows, white blood cells (WBC) count $2.5 \times 10^{9} / \mathrm{L}$ (normal 4-10×109/L); hemoglobin (Hb) level $8.4 \mathrm{~g} / \mathrm{L}$ (normal 12.0-15.0 g/L); platelet count $72 \times 10^{9} / \mathrm{L}$ (normal $150-410 \times 10^{9} / \mathrm{L}$ ); INR 1.32 (normal 1.0); total protein level $78.3 \mathrm{~g} / \mathrm{L}$ (normal 61-79 $\mathrm{g} / \mathrm{L}$ ); albumin level $51 \mathrm{~g} / \mathrm{L}$ (normal 35-48 g/L); alkaline phosphatase level $87 \mathrm{IU} / \mathrm{L}$ (normal 42$98 \mathrm{IU} / \mathrm{L})$; alanine transaminase level, $10 \mathrm{IU} / \mathrm{L}$ (normal 10-60 IU/L), and aspartate aminotransferase level, 20 IU/L (normal 10-42 IU/L).

In 2007 she was seen again by her hepatologist to stop the anticoagulant as she was planning to conceive. She had progressive pancytopenia at that time where she was referred to hematologist. Bone marrow for pancytopenia showed only $35 \%$ hypocellularity on trephine biopsy and peripheral blood test for PNH revealed deficient CD55 13.3\%, CD59 14.1\%. She required occasional blood transfusions after episodes of sever intravascular hemolysis with tea colored urine. A conventional treatment was given over 5 years in the form of anticoagulants, folic acid, oral iron supplements and blood transfusions. The laboratory results shown from 2006 up-to-date are summarized in Table 1.

In 2010 both her clinical and laboratory status deteriorated with severe pallor, lathery, feeling of sever fatigability shorting of breath on minimal exertion and hemoglobinuria. After meningococcal vaccination was given as a mandatory requirement before eculizumab treatment. The eculizumab was commenced on early 2011, where her clinical manifestations improved dramatically over three weeks. Although she was on high risk of thrombosis due to the BCS, portal vein stent and in spite of her need to continuous anticoagulant treat- ment, but she refused to take any anticoagulants since that time.

About three weeks after stating eculizumab treatment her general fatigability, shortening of breath and the hemoglobin level are all improved.

\section{Discussion}

Thrombosis is the main source of morbidity and mortality in $\mathrm{PNH}$; it occurs mostly in venous system in young patients without underlying atherosclerosis. In PNH the anticoagulants should be commenced quickly after diagnosis to prevent serious thrombosis, but unfortunately prophylactic anticoagulation is often dangerous to patients presenting with very low platelet counts. ${ }^{16}$ Studies have shown that there is an intrinsic relationship between the complement system and the coagulation cascade that is thought to cause thrombosis in PNH. Complement-mediated hemolysis, platelet activation, impairment of the fibrinolytic system, impaired NO bioavailability, and inflammatory mediators are all anticipated mechanisms which though to be responsible of increased thrombotic threat in PNH patients. The deficiency of CD59, makes platelets more susceptible to be attacked by complement, due to increased sensitivity to aggregation by thrombin, thrombin generation, and increased thrombotic risk, both in arterial and venous blood vessel. ${ }^{17}$ Activated platelets interact with neutrophils and could initiate thrombus formation by release of nucleosomes and neutrophil serine proteases, synergistically triggering Factor $\mathrm{X}$ further and thus activating blood coagulation mainly through the extrinsic pathway. Studies highlight decrease in thrombotic episodes after treating PNH patients with eculizumab (monoclonal antibody against the complement system). ${ }^{14,18}$ The antibody binds to the complement protein $\mathrm{C} 5$ and blocks its cleavage into $\mathrm{C} 5 \mathrm{a}$ and $\mathrm{C} 5 \mathrm{~b}$. Therefore, treatment with eculizumab prevents $\mathrm{C} 5 \mathrm{~b}$ formation, which is necessary to form MAC through binding to the complement proteins $\mathrm{C} 6, \mathrm{C} 7, \mathrm{C} 8$, and $\mathrm{C} 9$, which lead to thrombotic formation. ${ }^{19} \mathrm{BCS}$ is common in patients with $\mathrm{PNH}$, and anticoagulation therapy is the first choice for controlling this serious complication. Nevertheless, PNH patients often experience new thrombotic incidences despite sufficient anticoagulation. Thrombocytopenia is an additional PNH complication that occurs in 25-52\%. The anticoagulants therapy in the presence of low platelets predispose patient to high-risk of severe bleeding. The most adverse events reported for eculizumab treated $\mathrm{PNH}$ patients were nasopharyngitis, headache, back pain, and upper respiratory tract infections. ${ }^{20}$

\section{Conclusions}

Eculizumab treatment in this PNH patient showed protective antithrombotic action without the use of anticoagulants for more than four years. This protective measure is extremely valuable especially in thrombocytopenic PNH cases when the use of anticoagulants become seriously hazardous. Guidelines are needed after a randomized study to use eculizumab without anticoagulant agents, in order to avoid serious bleeding sequences in sever thrombocytopenic PNH patients.

\section{References}

1. Young N. Paroxysmal nocturnal hemoglobinuria and meyelodysplastic syndromes: clonal expansion of PIG-A-mutant hematopoietic cells in bone marrow failure. Haematologica 2009;94:3-7.

2. National Institute for Clinical Excellence. NICE Citizens Council Report Ultra Orphan Drugs. London: NICE; 2004.

3. Parker C, Omine M, Richards S, et al. Diagnosis and management of paroxysmal nocturnal hemoglobinuria. Blood 2005;106:3699-70.

4. Hong $\mathrm{Y}$, Ohishi $\mathrm{K}$, Inoue $\mathrm{N}$, et al. Requirement of N-glycan on GPI-anchored proteins for efficient binding of aerolysin but not Clostridium septicum $\alpha$-toxin. 
EMB0 J 2002;21:5047-56

5. Ferreira V, Pangburn M. Factor H-mediated cell surface protection from complement is critical for the survival of PNH erythrocytes. Blood 2007;110:2190-2.

6. Brodsky R. How I treat paroxysmal nocturnal hemoglobinuria. Blood 2009;113:65227.

7. Brodsky RA. Paroxysmal nocturnal hemoglobinuria: thephysiology of complementrelated hemolytic anemia. Ann Intern Med 2008;148:587-95.

8. Hill A Kell, RJ, Hillmen P. Thrombosis in paroxysmal nocturnal hemoglobinuria. Blood 2013;121:4985-96.

9. Ziakas P, Poulou L, Rokas GI, et al. Thrombosis in paroxysmal nocturnal hemoglobinuria: sites, risks, outcome. An overview. J Thromb Haemost 2007;5:642-5.

10. Brodsky R. Stem cell transplantation for paroxysmal nocturnal hemoglobinuria. Haematologica 2010;95:855-6.
11. Hall C, Richards S, Hillmen P. Primary prophylaxis with warfarin prevents thrombosis in paroxysmal nocturnal hemoglobinuria (PNH). Blood 2003;102:3587-91.

12. Meppiel E, Crassard I, Latour R, et al. Cerebral venous thrombosis in paroxysmal nocturnal hemoglobinuria: a series of 15 cases and review of literature. Medicine 2015;94:1-18.

13. Roth A, Duhrsen U. Paroxysmal nocturnal hemoglobinuria. Dtsch Arztebl 2007;104: 192-7.

14. Grunewald M, Grunewald A, Schmid A, et al. The platelet function defect of paroxysmal nocturnal haemoglobinuria. Platelets 2004;15:145-54.

15. Hillmen P, Muus P, Duhrsen U, et al. Effect of the complement inhibitor eculizumab on thromboembolism in patients with paroxysmal nocturnal hemoglobinuria. Blood 2007;110:4123-8.

16. Malato A, Saccullo G, Coco L, et al.
Thrombotic complications in paroxysmal nocturnal haemoglobinuria: a literature review. Blood Transfus 2012;10:428-35.

17. Moyo V, Mukhina G, Garrett E, Brodsky R. Natural history of paroxysmal nocturnal hemoglobinuria using modern diagnostic assays. Br J Haematol 2004;126:133-8.

18. Holada K, Simak J, Risitano AM, et al. Activated platelets of patients with paroxysmal nocturnal hemoglobinuria express cellular prion protein. Blood 2002;100:3413 .

19. Kelly R, Richard S, Hillmen P, Hill A. The pathophysiology of paroxysmal nocturnal hemoglobinuria and treatment with eculizumab. Ther Clin Risk Manag (Dovepress) 2009;5:911-21.

20. Hillmen P, Elebute M, Kelly R, et al. Longterm effect of the complement inhibitor eculizuman on kidney function in patients with paroxysmal nocturnal hemoglobinuria. Am J Hematol 2010;85:553-9. 